\title{
The art of measurement
}

\author{
With a dedicated monthly column, Nature Physics draws attention to metrology. And a set of \\ Commentaries in this issue focuses on various aspects of thermometry.
}

Physics is all about observations, quantified through measurements, expressed in units. The way our dealing with physical units has evolved is inevitably intertwined with our growing understanding of physics itself. For example, after the introduction of the Celsius scale (based on the freezing and boiling points of water), it was only a matter of time before the notion of absolute zero got sorted and the Kelvin scale was established.

Scientists worldwide now use the International System of Units (SI) ${ }^{1}$. It is all too easy to take this system for granted; defining and curating a consistent yet practical system of units is a taxing task. Maintaining and developing measurement standards is nowadays mostly done at national metrology institutes. Research at these and other metrology laboratories continues to move fundamental physics forward and spark new technologies. But the relevance of the SI and state-of-the-art measurement techniques goes far beyond scientific research: it pervades commerce and society as well. Without reliable clocks, safe navigation at sea was almost impossible ${ }^{2}$ and at present, high-frequency trading requires extremely precise and accurate timekeeping ${ }^{3}$.

There is a lot to be said about the units we use. Starting this month, Nature Physics features a column on metrology, in its broadest sense, replacing the science-fiction series Futures. (Fans of the latter need not worry - Nature will continue to publish weekly sci-fi bites at http://www.nature.com/ nature/focus/arts/futures.) These short one-page essays, covering physical units, fundamental constants or anything else measurement-related, are meant to be informative, yet at the same time accessible to the non-specialist. By making the series freely available, we hope to reach an audience well beyond our core readership. The pieces are commissioned, but we welcome suggestions for topics at naturephysics@nature.com. One more thing: we're calling our new column Measure for Measure, after Shakespeare's play. (Admittedly, the great playwright's inspiration was biblical, rather than metrological - let's say we'd like to suggest that there is an element of art to fine measurements.)
On page 104, Michael de Podesta kicks off Measure for Measure with a reflection on the kelvin.

Accompanying our new back page's inaugural instalment is a Focus on Thermometry consisting of four Commentaries exploring how we have come to define and understand temperature and how we've become accomplished in reaching and measuring it in a range spanning tens of $\mathrm{pK}$ to tens of MK.

The planned redefinition of the kelvin is only one of a few modifications to the SI to be ratified in 2018. As Joachim Fischer and Joachim Ullrich explain on page 4, all of the seven SI base units will ultimately be defined in terms of fundamental physical constants. To make this happen, metrologists are busy realizing measurements of the Boltzmann, Planck and Avogadro constants with ever higher accuracies.

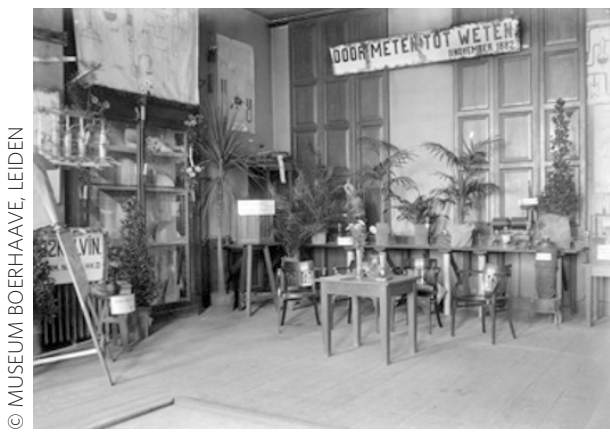

Most of the thermometry efforts leading to the redefinition of the kelvin specifically, accurate measurements of the Boltzmann constant - take place in the 'moderate' temperature range (1-1,235 K). On page 7, Michael Moldover and co-authors give an overview of the progress made in this field. With various methods, relying on different physical principles, impressive accuracies can now be achieved, down to a few parts per million. In fact, accuracies now have become such that 'errors' in the International Temperature Scale of 1990 have been uncovered.

Many physicists, when contemplating the concept of temperature, will no doubt think of low temperatures - because quantum effects are often associated with low $T$. (It is worth pointing out, though, that quantum signatures can also be seen at room temperature; the quantum Hall effect in graphene is a spectacular example ${ }^{4}$.) As Juha Tuoriniemi discusses on page 11, our desire to reach ever lower temperatures has led to many scientific and technological breakthroughs.

Helium-dilution refrigerators can now continuously maintain temperatures of a few $\mathrm{mK}$ and some systems can be magnetically cooled to below $100 \mathrm{pK}$. Meanwhile, cryogenics has become a vast industry and cryostats are now a fixture in labs all over the world. Major physics discoveries at low temperature are numerous: superconductivity, superfluidity and Bose-Einstein condensation arguably make up the top three. The Cold Atom Lab, scheduled to be deployed at the International Space Station later this year (http://coldatomlab.jpl.nasa.gov/) with the aim of realizing the "coolest spot in the Universe", is yet another exponent of our never-ending quest for lower and lower temperatures.

The other extreme, ultra-high temperatures, is pursued in nuclear fusion experiments, mimicking the energy production processes taking place in the Sun. As Didier Mazon and co-authors explain on page 14 , heating a mixture of deuterium and tritium ions to more than 150 million degrees Celsius is difficult, but achievable. One of the breakthroughs in this context is the tokamak, essentially a torus-shaped vessel dressed in a complex configuration of coils through which electric currents are driven to generate magnetic fields that confine plasma ions and electrons. A huge internationally funded tokamak, ITER (https://www.iter.org/mach), is being built in the south of France with the purpose of demonstrating the feasibility of energy production from nuclear fusion.

It's clear that progress in metrology and advances in science go hand in hand. Heike Kamerlingh Onnes's laboratory motto (pictured) captures it nicely: door meten tot weten - through measuring to knowing.

References

1. International System of Units (SI) 8th edn (BIPM, 2014); http://www.bipm.org/en/publications/si-brochure/

2. Sobel, D. Longitude (Fourth Estate, 1996).

3. Buchanan, M. Physics in finance: Trading at the speed of light. Nature News (11 February 2015); http://go.nature.com/y34Zav

4. Novoselov, K. S. et al. Science 315, 1379 (2007). 